\title{
Electrochemical Behavior of 1,2,4-Triazole and Benzotriazole at Glassy Carbon Electrode in Acidic Media
}

\author{
S.V. Lokesh ${ }^{1}$, A.K. Satpati ${ }^{2, *}$ and B.S. Sherigara ${ }^{1, *}$ \\ ${ }^{I}$ Department of PG Studies and Research in Industrial Chemistry, Jnana Sahyadri, Shankaraghatta, Shimoga, \\ Karnataka- 577 301, India \\ ${ }^{2}$ Analytical Chemistry Division, Bhabha Atomic Research Centre, Mumbai-400085, India
}

\begin{abstract}
Electrochemical investigations of 1,2,4-triazole and benzotriazole in aqueous acidic media has been carried out by cyclic voltammetric measurements. Cyclic voltammetric experiments were carried on glassy carbon (GC) working electrode. Effect of potential scan rate, $\mathrm{pH}$ of the solution and use of different surfactant on the electrochemical behavior has been investigated. Single reduction wave was observed for 1,2,4-triazole due to the reduction of N=N moiety and no oxidation peak was observed in the reverse scan. In case of benzotriazole one clear reduction peak was observed in the cathodic scan and no anodic peak was seen. Effect of $\mathrm{pH}$, sweep rate, analyte concentrations and the presence of different surfactants on the reduction peak current was evaluated. Electro-reduction processes of both the compounds were found to be diffusion controlled in nature. The plausible mechanism for the electroreduction process was proposed. Electrochemical property of two compounds was also studied under hydrodynamic conditions using glassy carbon rotating disc electrode.
\end{abstract}

Keywords: 1-(2-pyridylazo)-2-naphthol, Benzotriazole, Voltammetry, Rotating Disc Electrode (RDE).

\section{INTRODUCTION}

Use of pesticides in agricultural production and the negative environmental impacts associated with these compounds has led to the development of different electroanalytical techniques [1-7] and studying the electrokinetics of such compounds. Electrochemical methods are applied in toxicological, ecotoxicological and environmental regulations due to the applicability over a wide concentration range [8] using techniques starting from coulometry, cyclic voltammetry to differential pulse voltammetry.

The basic building block of the compound, 1,2,4-Triazole has got very wide varieties of applications, most notably, it is used as antifungal in the form of fluconazole and itraconazole. There are many derivatives of triazole used as the corrosion inhibitor in the corrosion inhibition of steel and copper [9-11]. In some of our recent reports we have used benzotriazole as the corrosion inhibitor in acidic media [10, 11] and the mechanism of the inhibition have been reported. Electrosorption and polymerization of triazoles at the electrode-electrolyte interface using brass and gold electrode have been discussed in some of the recent studies [12-14]. Triazoles can present in different tautomeric forms, effect of such tautomeric forms on the electrochemical property of

*Address correspondence to these authors at the Department of PG Studies and Research in Industrial Chemistry, Jnana Sahyadri, Shankaraghatta, Shimoga, Karnataka- 577 301, India; Tel: +91-22-25590326;

Fax: 91-22-25505151, E-mail: asatpati@barc.gov.in and Analytical Chemistry Division, Bhabha Atomic Research Centre, Mumbai-400085, India; Tel: +91 08282 256222; Fax: +91 08282 256255;

Email: bssherigara@rediffmail.com triazoles. On gold electrode surface triazole produces two redox peaks, the pre-peak before the diffusion controlled peak was attributed to be due to the adsorption of triazole on gold surface.

In the present study the two compounds studied are belonging to the triazole group and they are used for many similar purposes. The chemical moiety of the two compounds are same (triazole ring) but the chemical identity of the two compounds are differed by the introduction of a benzene ring in benzotriazole, which is absent in triazole. Therefore it is very interesting and important to see the redox behavior of 1,2,4-triazole and benzotriazole in aqueous media and compare their behavior. In the present study, electrochemical property of two triazoles has been studied in aqueous solution using glassy carbon as the working electrode. Effect of $\mathrm{pH}$, sweep rate and effect of different surfactants on the reduction process of the two compounds has been investigated. Electroreduction behavior has also been investigated under hydrodynamic conditions using rotating disc electrode.

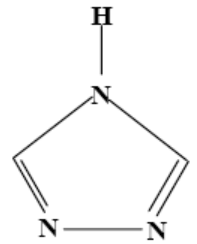

(A)

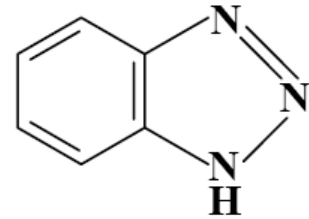

(B)
Chart 1 Chemical structure of (A) 1,2,4 triazole and (B) benzotriazole.

2010 Bentham Open 


\section{EXPERIMENTAL}

\subsection{Reagents and Chemicals}

Triazoles were purchased from Merck and used as obtained. Cetyl trimethyl ammonium bromide (CTAB), sodium dodecyl sulphate (SDS) and Triton X-100 were obtained from Merck and used without further purification. All other reagents used in the present study were of analytical reagent grade. All solutions were prepared using double distilled and de-ionized water. Britton-Robinson buffer was used to adjust the $\mathrm{pH}$ of the supporting electrolyte solution at the wide range of $\mathrm{pH}$ 's from 2 to 12 . It was prepared by mixing 1:1:1 ratio of $0.04 \mathrm{M}$ boric acid $\left(\mathrm{H}_{3} \mathrm{BO}_{3}\right)$, phosphoric acid $\left(\mathrm{H}_{3} \mathrm{PO}_{4}\right)$ and acetic acid $\left(\mathrm{CH}_{3} \mathrm{COOH}\right)$.

\subsection{Instrumentation and Apparatus}

Cyclic voltammetric experiments were carried out using a three-electrode configuration consisting of a glassy carbon (disk, $3 \mathrm{~mm}$ diameter) as a working, Pt foil as the counter and saturated calomel electrode (SCE) as the reference electrode. All potentials reported are related to this reference electrode. The electrochemical measurements were performed using an Eco Chemie make Potentiostat/Galvanostat Autolab-100 and the analysis was made by GPES- 4.9 software. The glassy carbon electrode was polished with $0.3 \mu \mathrm{m}$ alumina powder and then rinsed with distilled water before use. All the experiments were performed at room temperature. Prior to the electrochemical scan solutions were purged with nitrogen for $15 \mathrm{~min}$. In cyclic voltammetric measurements the applied potential was scanned from $0 \mathrm{~V}$ to $-1.6 \mathrm{~V}$ with respect to SCE. Rotating disc electrode (RDE) experiments were carried out using the PINE electrode assembly with pine speed control system connected to the AUTOLAB 100 potentiostat. The RDE used in this investigation was carried out using the glassy carbon as the working electrode attached with the PINE electrode assembly. In all the tables the spread in the data points were presented as the 2 sigma (2s) from 3 repeated current measurements.

\section{RESULTS AND DISCUSSION}

Cyclic voltammetric scans of 1,2,4-triazole at a concentration of $2 \times 10^{-3} \mathrm{M}$ in aqueous acid media using $\mathrm{HCl}$ as the supporting electrolyte, with a sweep rate of $0.05 \mathrm{mV} \mathrm{s}^{-1}$ at glassy carbon electrode was obtained and shown in (Fig. 1). In case of 1,2,4-triazole, a single cathodic wave and no anodic peak was appeared at the experimental potential window. From the chemical structure and cyclic voltammograms of the investigated compounds it is indicated that the peak at around $-1.5 \mathrm{~V}$ vs. SCE was due to reduction of azo group present in the molecule. In case of benzotriazole one reduction peak was obtained at $-1.48 \mathrm{~V}$ and no anodic peak in the reverse scan was seen (Fig. 2).

\subsection{Effect of the Concentration of the Compounds}

The observed reduction peak current was found to increase linearly with the concentration of triazole at the concentration range of $0.5 \times 10^{-3}$ to $4 \times 10^{-3} \mathrm{M}$ in $50 \times 10^{-3} \mathrm{M}$ in $\mathrm{HCl}$ solution as shown in the Table $\mathbf{1}$. The scan rate was kept at $0.05 \mathrm{Vs}^{-1}$ for this study of obtaining the concentration dependence on the reduction peak current. This linear increase in the reduction current with concentration indicates the elec-

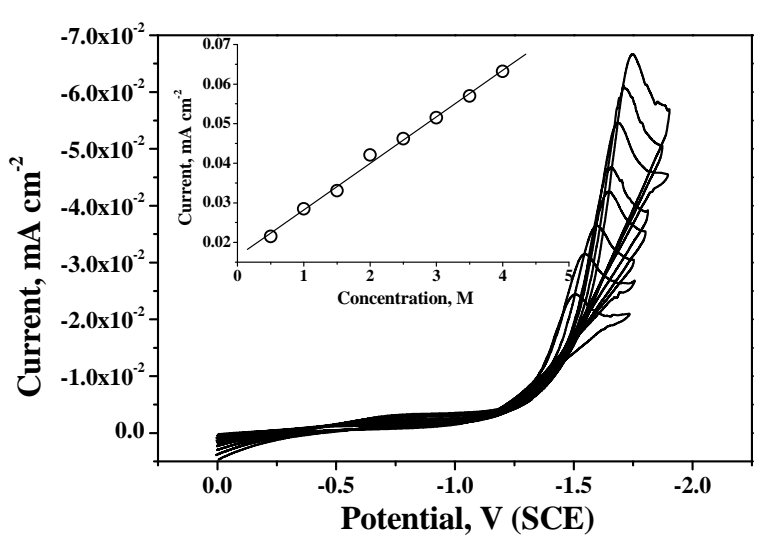

Fig. (1). Cyclic voltammogram of $1 \mathrm{mM}$ 1,2,4-triazole at glassy carbon electrode in aqueous acidic media, $\mathrm{pH}=3.1$, Inset: Plot of concentration versus cathodic peak current on reduction of $1,2,4$ triazole at GCE.

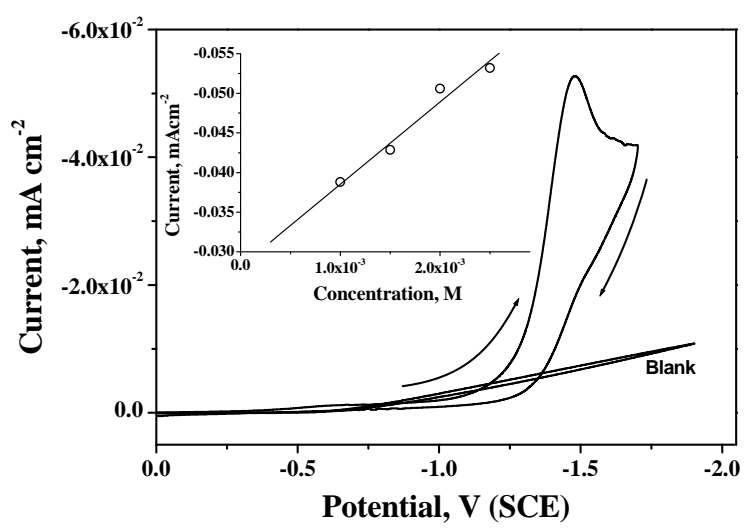

Fig. (2). Cyclic voltammogram of $1 \mathrm{mM}$ benzotriazole at glassy carbon electrode in aqueous acidic media $\mathrm{pH}=2.85$, Cyclic voltammogram of the blank solution is also shown. Inset: Plot of concentration versus cathodic peak current of the peak on reduction of benzotriazole at GCE.

Table 1. The Change in the Peak Potential and the Peak Current of 1,2,4-Triazole with the Change in Concentration

\begin{tabular}{|c|c|c|}
\hline $\begin{array}{c}\text { 1,2,4-Triazole } \\
\begin{array}{c}\text { Concentration } \\
\mathbf{~ m M}\end{array}\end{array}$ & $\begin{array}{c}\text { Potential } \mathbf{E}_{\mathbf{p c}}(\mathbf{V}) \\
\mathbf{\pm 0 . 0 2}(\mathbf{V})\end{array}$ & $\begin{array}{c}\text { Current } \mathbf{i}_{\mathbf{p c}}\left(\mathbf{m A c m}^{-2}\right) \\
\mathbf{\pm 0 . 0 0 5}\left(\mathbf{m A ~ \mathbf { ~ m } ^ { - 2 }}\right)\end{array}$ \\
\hline \hline 0.5 & -1.51 & -0.034 \\
\hline 1.0 & -1.54 & -0.037 \\
\hline 1.5 & -1.60 & -0.039 \\
\hline 2.0 & -1.65 & -0.047 \\
\hline 2.5 & -1.66 & -0.052 \\
\hline 3.0 & -1.70 & -0.057 \\
\hline 3.5 & -1.72 & -0.059 \\
\hline 4.0 & -1.75 & -0.062 \\
\hline
\end{tabular}

troreduction process to be diffusion controlled in nature. The peak current value of triazole ( $c f$. Fig. 1) follows the regression equation of $\mathrm{I}=0.1644+0.1175 \mathrm{C}$ with standard devia- 
tion (SD) of 0.109 and correlation coefficient $\mathrm{R}=0.995$. Here the current, I is expressed in $\mathrm{mAcm}^{-2}$ and concentration, $\mathrm{C}$ in $\mathrm{mML}^{-1}$ unit. The peak potential was found to be shifted from $-1.5 \mathrm{~V}$ to $-1.75 \mathrm{~V}$ with the addition of higher concentration of triazole. The change in peak potential with the addition of analyte concentration indicates irreversible nature of the electrochemical process. There can also be the possibility of aggregation of the triazole molecules at higher concentration, which may lead to the shifting of the potentials to more negative values.

In case for benzotriazole the peak current values increase linearly with the concentration as shown in the inset of Fig. 2 and the data points were shown in Table 2 . The peak current values of benzotriazole ( $c f$. Fig. 2B) follow the regression equation of $\mathrm{I}=0.281+0.105 \mathrm{C}$ with standard deviation (SD) of 0.123 and correlation coefficient $\mathrm{R}=0.992$. Here the current, $\mathrm{I}$ is expressed in $\mathrm{mAcm}^{-2}$ and concentration, $\mathrm{C}$ in $\mathrm{mML}^{-}$ ${ }^{1}$ unit. The peak potential of benzotriazole does not shift any significantly with the increase in the concentration of benzotriazole.

Table 2. The Change in the Peak Potential and the Peak Current of Benzotriazole with the Change in Concentration

\begin{tabular}{|c|c|c|}
\hline Benzotriazole & \multicolumn{2}{|c|}{ Reduction Peak } \\
\hline $\begin{array}{c}\text { Concentration } \\
\mathbf{m M} / \mathbf{L}\end{array}$ & $\begin{array}{c}\text { Potential } \mathbf{E}_{\mathbf{p c}}(\mathbf{V}) \\
\mathbf{\pm 0 . 0 2}(\mathbf{V})\end{array}$ & $\begin{array}{c}\text { Current } \mathbf{i}_{\mathbf{p c}}\left(\mathbf{m A c m}^{-2}\right) \\
\mathbf{\pm 0 . 0 0 5}\left(\mathbf{m A ~ c m}^{-2}\right)\end{array}$ \\
\hline \hline 0.5 & -1.48 & -0.033 \\
\hline 1.0 & -1.48 & -0.039 \\
\hline 1.5 & -1.49 & -0.041 \\
\hline 2.0 & -1.49 & -0.051 \\
\hline 2.5 & -1.49 & -0.053 \\
\hline
\end{tabular}

When the electrochemical behavior of two compounds were compared it was observed that the peak potential undergo a cathodic shift in case of 1,2,4-triazole with increase in the concentration, the peak potential of benzotriazole remains unchanged. The shift in the peak potential is indicative of the irreversible nature of the electro-reduction process of 1,2,4-triazole, however, there is also a possibility of intermolecular interaction between triazole molecules, which might shift the peak potential towards more cathodic potentials. Similar type of intermolecular interaction as that in triazoles is minimized in case of benzotriazole due to the presence of a benzene ring, which might repel each other and oppose any kind of intermolecular interaction. Additionally benzotriazole being aromatic compound is expected to be more stable compared to 1,2,4-triazole so the comparative possibility of polymerization would be less in benzotriazole.

\subsection{Effect of Scan Rate}

The effect of scan rates on the electrochemical behavior of 1,2,4-triazole and benzotriazole has been studied in aqueous-hydrochloric acid media. The peak currents were measured at a varying scan rates from $10-500 \mathrm{mV} \mathrm{s}^{-1}$, the results are tabulated in Table 3. It is observed that in the case of 1,2,4-triazole the peak current increases with increase in the potential scan rates. The peak current values when plotted with respect to the square root of scan rates, results in a straight line plot as seen in (Fig. 3), which indicate that the process is diffusion controlled in nature $[15,16]$. The data points were fitted using Randle Sevcik's equation as.

Table 3. Effect of Scan Rate on Reduction of 1,2,4-Triazole at Glassy Carbon Electrode, $\mathbf{p H}=3.1$

\begin{tabular}{|c|c|c|}
\hline 1,2,4-Triazole & \multicolumn{2}{|c|}{ Reduction Peak } \\
\hline Scan Rate & $\begin{array}{c}\text { Potential-E } \\
\mathbf{p c} \\
\mathbf{\pm 0 . 0 2}(\mathbf{V})\end{array}$ & $\begin{array}{c}\text { Current } \mathbf{i}_{\mathbf{p c}}\left(\mathbf{m A ~ c m} \mathbf{~ c m}^{-2}\right) \\
\mathbf{\pm 0 . 0 0 5}\left(\mathbf{m A ~ c m} \mathbf{~ c m}^{-2}\right.\end{array}$ \\
\hline \hline 50 & -1.54 & -0.038 \\
\hline 100 & -1.54 & -0.042 \\
\hline 200 & -1.57 & -0.050 \\
\hline 300 & -1.59 & -0.059 \\
\hline 400 & -1.62 & -0.069 \\
\hline 500 & -1.64 & -0.072 \\
\hline
\end{tabular}

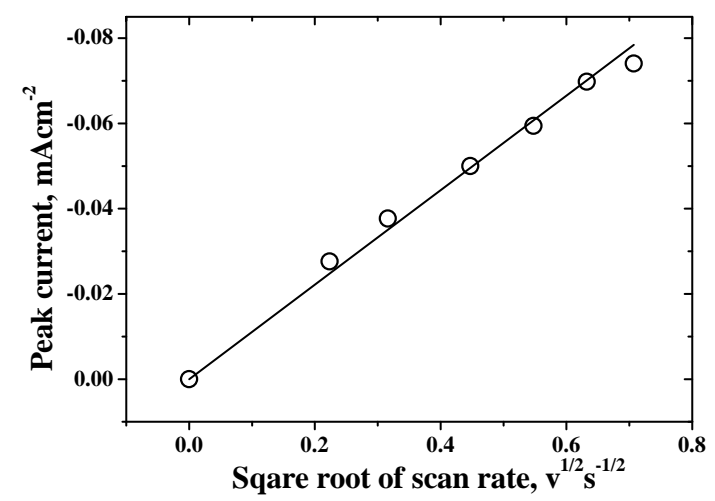

Fig. (3). Plot of square root of scan rate verses peak current on reduction of $1 \mathrm{mM}$ 1,2,4-triazole at $\mathrm{pH} 3.1$ on GCE.

$$
I_{p}=\left(2.69 \times 10^{5}\right) n^{3 / 2} A D^{1 / 2} v^{1 / 2} C
$$

where $I_{p}$ is the peak current in Amp, $n$ is the number of electron, $\mathrm{A}$ is the area of the electrode in $\mathrm{cm}^{2}, \mathrm{D}$ is the diffusion coefficient of the species in $\mathrm{cm}^{2} \mathrm{~s}^{-1}, v$ is the scan rate in $\mathrm{Vs}^{-1}$ and $\mathrm{C}$ is concentration of the species in $\mathrm{Mcm}^{-3}$. The constant $2.69 \times 10^{5}$ was obtained from the numerical relation between $\left(E-E_{1 / 2}\right) n$ and $\sqrt{\pi \chi}(\sigma t)$ for a reversible electrochemical process with $\left(E-E_{1 / 2}\right) n$ value of $28.50 \mathrm{mV}$ at $25^{\circ} \mathrm{C}$ [15]. It is observed that the experimental data points are fitted well with the straight line equation passing through origin and the with correlation coefficient $\mathrm{r}$ as 0.996 . Thus, the electroreduction process of 1,2,4-triazole is found to be diffusion controlled in nature. The reduction peak potential shifted from $-1.54 \mathrm{~V}$ to $-1.64 \mathrm{~V}$ with increase in the scan rates, which indicates the irreversible nature of the electrochemical process. For the reactions that are 'slow' (so called quasi- 
reversible or irreversible electron transfer reactions) the voltage applied does result in the generation of the concentrations at the electrode surface predicted by the Nernst equation. This happens because the kinetics of the reaction is 'slow' and thus the equilibrium is not established rapidly as comparison to the voltage scan rate. In this kind of situation the overall form of the voltammogram remains similar to the reversible system but position of the current maximum, peak potential shifts depending upon the reduction rate constant and also the applied voltage scan rates. This occurs because the current takes more time to respond to the applied voltage than the reversible case [15].

Similarly in case of benzotriazole the cathodic peak current increases with the increase in the scan rates. The peak current values were found to be proportional to the square root of the scan rate and also follow the equation.1 (plots not shown) thereby indicating the diffusion controlled electrochemical process.

\subsection{Effect of $\mathbf{p H}$}

Effect of $\mathrm{pH}$ on the reduction behavior of 1,2,4-triazole and benzotriazole was studied by varying the $\mathrm{pH}$ from 2 to 9.2 using B-R buffer medium. In case of 1,2,4-triazole as the $\mathrm{pH}$ increases from 2 to 9.2 , the cathodic peak potential was found to shift towards more negative values and also a slight decrease in peak current was observed, which confirms that the 1,2,4-triazole is more electrochemically active at $\mathrm{pH} 3.1$ (Fig. 4A). Statistical information in the experimental result was obtained from 5 independent measurements and 2 times of the standard deviation (2s) was presented as the error bar in the plot. The peak current also decreased drastically above $\mathrm{pH} 3.15$ and no peak was observed above $\mathrm{pH} 4$. This implies that as the $\mathrm{pH}$ increases the reduction becomes more difficult which indicates involvement of proton in the reduction process and the reduction of both molecules is very sensitive to the $\mathrm{pH}$ of the medium. Similarly in case of benzotriazole, as seen from (Fig. 4B) the peak current remains high at $\mathrm{pH}$ up to 2.8 , beyond that the peak current decreases sharply. Therefore the observations indicate that $\mathrm{pH}$ has a very significant role in the electroreduction process of both 1,2,4triazole and benzotriazole. Based on the present observation and reported literature a scheme of the electroreduction process of 1,2,4-triazole has been proposed [17] which, is presented in Chart 2.

\subsection{Electrochemical Behavior in Surfactant Media}

Keeping the concentration of 1,2,4-triazole at $1 \times 10^{-3} \mathrm{M}$, pH 2.3 in B-R buffer with a scan rate $50 \mathrm{mVs}^{-1}$, the voltammograms were recorded by increasing the concentration of three different surfactants from $1 \times 10^{-5} \mathrm{M}$ to $6 \times 10^{-5} \mathrm{M}$. The observed change in the current with increase in the surfactant concentration is shown in (Fig. 5).

In the case of anionic surfactant, sodium dodecyl sulfate (SDS), the peak current decreases with increase in the addition of SDS and peak potentials shifted to the negative direction. Different factors affect the surfactant adsorption on solid/liquid interface [15]. Apart from this, it is also claimed that at higher concentrations of SDS, the repulsive forces among the surfactant molecules adsorbed at the interface of the solid (adsorbent)-solution are more effective [18]. By

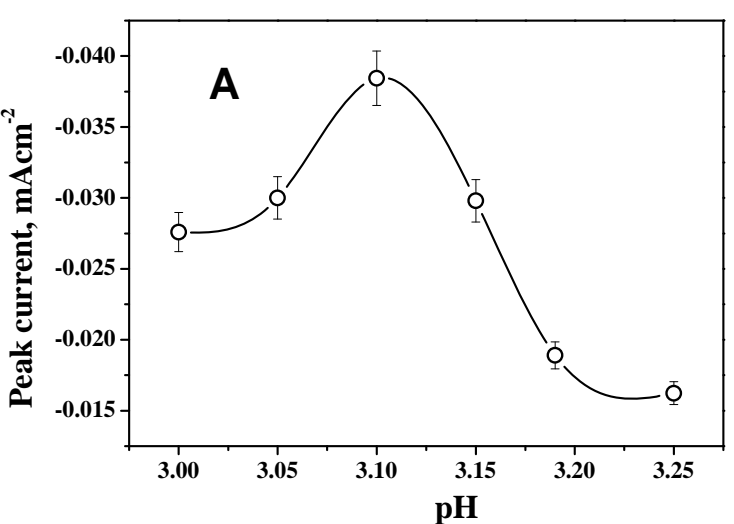

Fig. (4A). Plot of $\mathrm{pH}$ verses peak current on reduction of $1 \mathrm{mM}$ 1,2,4-triazole at GCE.

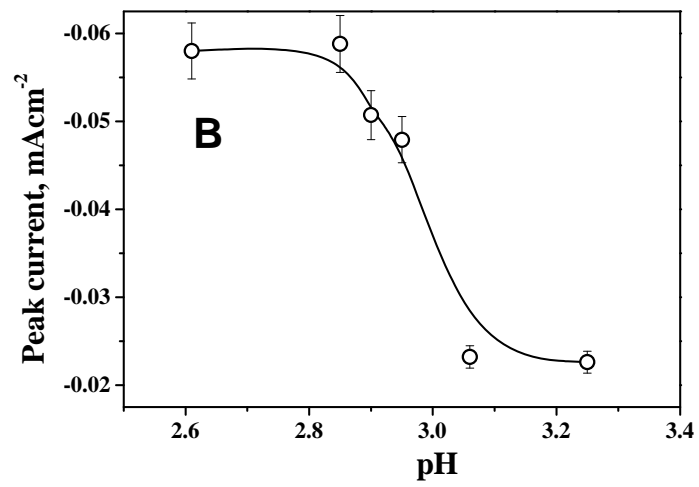

Fig. (4B). Plot of $\mathrm{pH}$ versus cathodic peak current on reduction of $1 \mathrm{mM}$ benzotriazole at GCE.

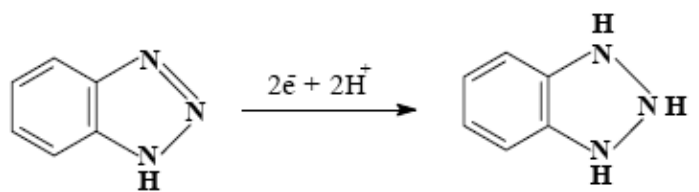

Chart 2 Proposed scheme of the electroreduction process of benzotriazole.

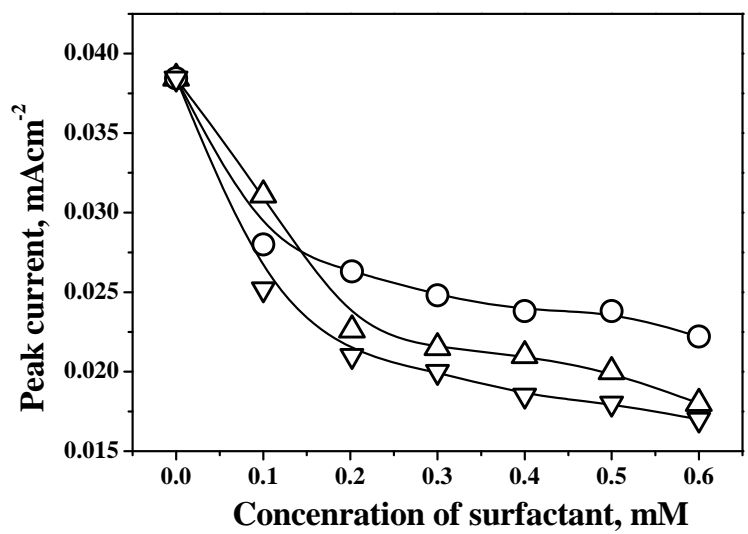

Fig. (5). Effect of different surfactants on the voltammetric peak of $1 \mathrm{mM} 1,2,4$ triazole $(\mathrm{O}) \mathrm{TX} 100,(\Delta)$ CTAB $(\nabla)$ SDS on GCE at $\mathrm{pH}$ 3.1 .

increasing the concentration of the surfactant, more and more surfactant gets adsorbed at the surface of the electrode resulting in the decrease in the cathodic current and also the peak 
Table 4. Effect of Variation of Surfactants with 1,2,4-Tiazole, Scan Rate: $50 \mathrm{mVs}^{-1}$ Concentration of the Compound $1 \times 10^{-3} \mathrm{M}$. The 2 Sigma Spread in Potential is $\pm 0.02(\mathrm{~V})$, and to the Current is $\pm 0.005\left(\mathrm{~mA} \mathrm{~cm}^{-2}\right)$

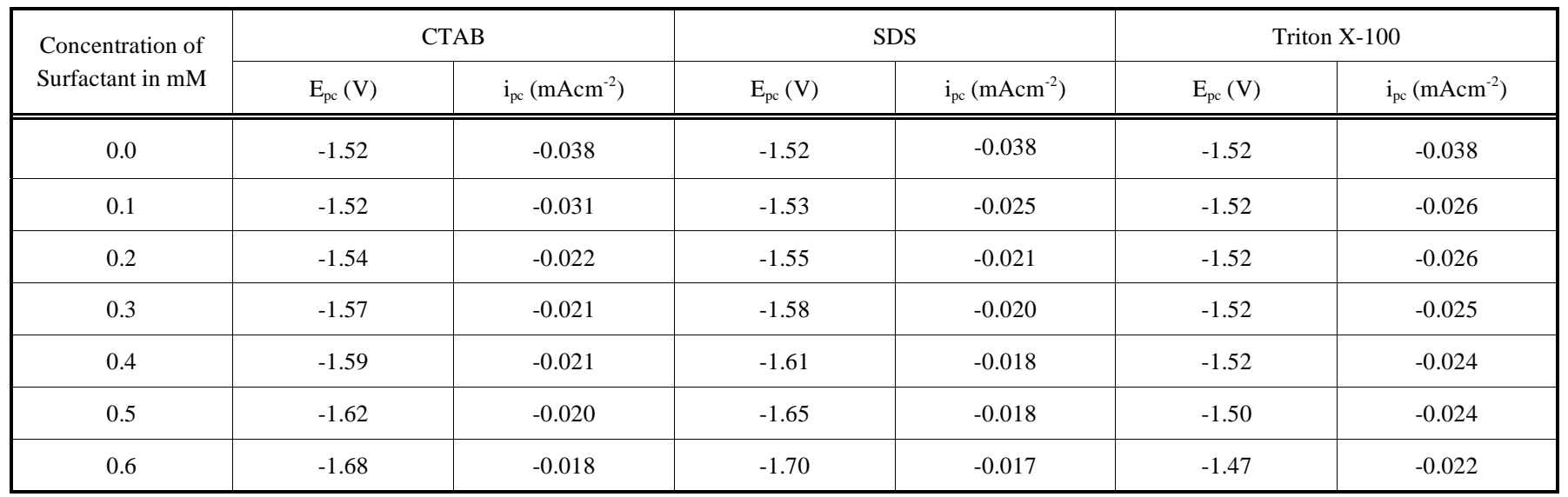

potential was shifted towards more negative direction. This might be due to the change in the heterogeneous kinetics of the electron transfer process between the electrode surface and molecules in the interfacial region affected by the adsorbed surfactant materials as the microstructure and the active sites on the electrode surface were damaged.

In the case of cationic surfactant CTAB, the peak potential does not change with the increase in concentration of CTAB (up to $6.0 \times 10^{-5} \mathrm{M}$ ) but the peak current decreases as the concentration of CTAB increases. The decrease in the peak current could be due to the slower diffusion of the electroactive species as a result of higher viscosity of the medium. The cathodic peak was not observed at the higher concentration of CTAB. This indicates the saturation capacity [19] of the electrode for adsorption of the cationic surfactant due to the electrostatic interaction between the adsorbent and the surfactant, the decrease in current values can be seen from Table 4. The nonionic surfactant Triton X-100 affects the electrochemical behavior of 1,2,4-triazole similar to that of CTAB. The peak potential, $\mathrm{E}_{\mathrm{pc}}$, remains unchanged but the peak current, $\mathrm{i}_{\mathrm{pa}}$ decreased with increase in concentration of TX100.

\subsection{Rotating Disc Electrode (RDE) Study}

RDE experiments were carried out using the PINE electrode assembly with pine speed control system connected to the AUTOLAB 100 potentiostat. Voltammograms were recorded with varying rotation speed from $500 \mathrm{rpm}$ to 2500 $\mathrm{rpm}$. The saturation in the reduction current is seen beyond $1.6 \mathrm{~V}$ in both the cases however with increase in the rotation speed there is a shift in the potential towards the negative direction ( $c f$ Fig. 6A and 6B). The limiting current value increases with increase in the rotation speed. Variation in the limiting current and the corresponding potential with the change in rotation speed for 1,2,4 triazole and benzotriazole are reported in Table 5 and Table 6 respectively.

Plot of the limiting current with respect to the angular rotation frequency is plotted in (Fig. 7A and 7B). The data points were found to fit well with linear function passing through the origin. Therefore the correlation between the limiting current density $\left(i_{1}\right)$ with the angular frequency $\omega$ found to follow the Levich relation as;

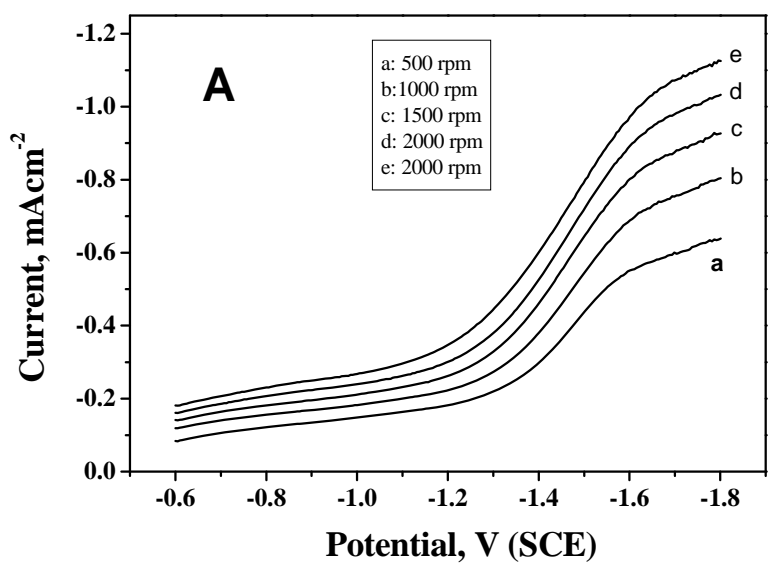

Fig. (6A). Voltammogram of $1 \mathrm{mM}$ 1,2,4-triazole under hydrodynamic conditions on GCE with different rotation speed. Solution $\mathrm{pH}$ was maintained at 3.1.

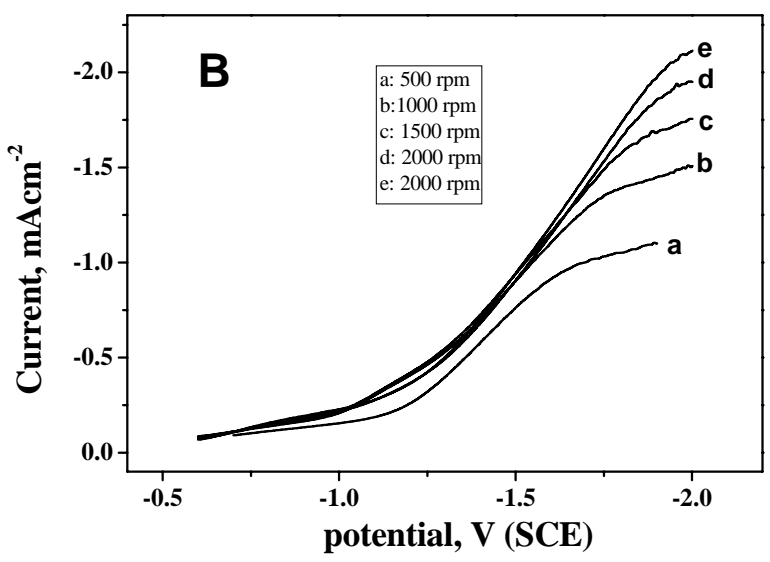

Fig. (6B). Voltammogram of $1 \mathrm{mM}$ benzotriazole under hydrodynamic conditions with different rotation speed on GCE. Solution $\mathrm{pH}$ was maintained at 2.85 .

$\mathrm{i}_{1}=0.62 \mathrm{n} \mathrm{FA} \mathrm{D}^{2 / 3} v^{-1 / 6} \omega^{1 / 2} \mathrm{C}$

where $\mathrm{n}$ is the number of electron, $\mathrm{F}$ is Faraday constant, D is the diffusion coefficient in $\mathrm{cm}^{2} \mathrm{~s}^{-1}, v$ is the kinematic viscosity (i.e. , viscosity of the solution divided by its density) in this case it is considered to be $0.01 \mathrm{~cm}^{2} \mathrm{~s}^{-1}$. The constant 
Table 5. RDE Results of 1,2,4-Triazole Scan Rate $50 \mathrm{mVs}^{-1}$

\begin{tabular}{|c|c|}
\hline Rotation speed (rpm) & Current, $\mathbf{i}_{\mathbf{l}}\left(\mathbf{m A c m}{ }^{-2}\right) \mathbf{\pm 0 . 0 6}\left(\mathbf{m A ~ c m}^{-2}\right)$ \\
\hline \hline 500 & -0.54 \\
\hline 1000 & -0.68 \\
\hline 1500 & -0.81 \\
\hline 2000 & -0.92 \\
\hline 2500 & -1.05 \\
\hline
\end{tabular}

Table 6. RDE Results of 1,2,4-Triazole Scan Rate $50 \mathrm{mV} / \mathrm{s}$

\begin{tabular}{|c|c|}
\hline Rotation Speed (rpm) & Current, $\mathbf{i}_{\mathbf{l}}\left(\mathbf{m A ~ c m} \mathbf{~ c}^{-2}\right) \mathbf{0 . 0 6}\left(\mathbf{m A ~ c m}^{-\mathbf{2}}\right)$ \\
\hline \hline 500 & -0.97 \\
\hline 1000 & -1.37 \\
\hline 1500 & -1.64 \\
\hline 2000 & -1.85 \\
\hline 2500 & -2.06 \\
\hline
\end{tabular}

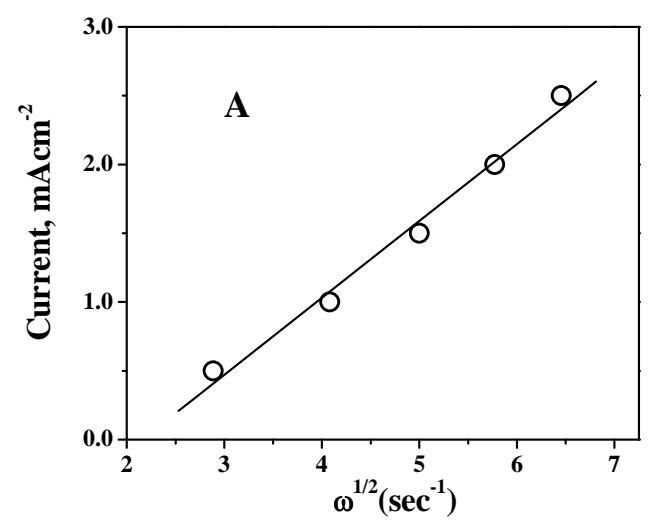

Fig. (7A). Levich plot of 1,2,4 triazole for hydrodynamic voltammetry.

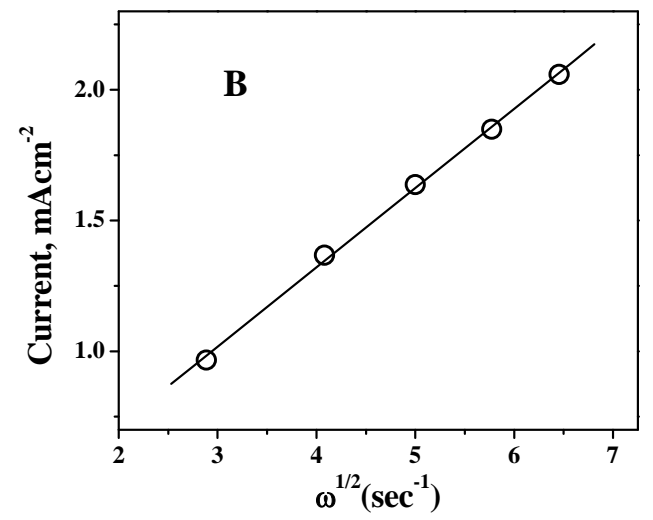

Fig. (7B). Levich plot of benzotriazole for hydrodynamic voltammetry.

0.62 is the Levich constant for totally mass transfer-limited conditions at the rotating disc electrode [15]. Using the
Levich equation the diffusion coefficient for 1,2,4-triazole is obtained as $19.1 \times 10^{-6} \mathrm{~cm}^{2} \mathrm{~s}^{-1}$ and for benzotriazole it is obtained as $7.5 \times 10^{-6} \mathrm{~cm}^{2} \mathrm{~s}^{-1}$. The diffusion coefficient of triazole is found to be higher than benzotriazole; this is due to smaller size of triazole compared to benzotriazole.

Interestingly it was observed that the hydrodynamic voltammogram in case of benzotriazole was comparatively steeper than 1,2,4-triazole. Benzotriazole is a bulkier molecule than 1,2,4-triazole therefore with increase in rotation speed the change in the current is more pronounced in benzotriazole than 1,2,4-traizole which was resulted the steeper hydrodynamic voltammogram. Additionally it was also observed that the half-wave potentials of the hydrodynamic voltammograms were found to be more positive in the both benzotriazole and 1,2,4 triazole than the peak potential of the cyclic voltammogram obtained under static conditions. This might be due to the lesser possibility of adsorption of triazole species at the electrode surface under hydrodynamic conditions which otherwise present under static conditions resulted in the increased surface resistance and peak shifting towards negative direction.

\section{CONCLUSION}

Cyclic voltammetric investigations were carried out for 1,2,4-triazole and benzotriazole. Peak potential was found to shift to more positive value with increase in the acidity of the medium, indicating easier reduction due to the involvement of proton in the reduction process. Cathodic peak current was found to increase linearly with square root of sweep rate and also with concentration of electroactive species. This suggests that overall electrode process is diffusion controlled and irreversible in nature. Diffusion coefficients of 1,2,4triazole and benzotriazole were calculated from the RDE voltammetry.

\section{REFERENCES}

[1] Mendez, J. H.; Martinez, R. C.; Gonzalo, E. R. Electroanalytical study of the pesticide guthion. J. Electroanal. Chem., 1988, 244, 221-233.

[2] Li, C.; James, B. D.; Magee, R. J. The determination of organochlorine compounds by adsorptive stripping voltammetry, Part I:p,p'-DDT and dieldrin. Electroanalysis, 1990, 2, 63-67.

[3] Mendez , J. H.; Martinez, R. C.; Gonzalo, E. R. Analytical and mechanistic aspects of the polarographic reduction of the herbicide pyrazon. Electroanalysis, 1990, 2, 389-396.

[4] Martinez, R. C.; Becerra, F.; Mendez, J. H.; Martin, P. G. Electroanalytical determination of diazinon: direct current and differential pulse polarography and adsorptive stripping voltammetry. Electroanalysis, 1990, 2, 567-571.

[5] Smyth, M. R.; Osteryoung, J. G. A pulse polarographic investigation of parathion and some other nitro-containing pesticides. Anal. Chim. Acta., 1978, 96, 335-344.

[6] Nascimento, P. C. Do; Bohrer, D.; Carvalho L. M. de; Trevisan J.; Eduardo J. P.; Vendrame, Z. B.; Dessuy, M. B. Determination of triazines in hemodialysis saline solutions by adsorptive stripping voltammetry after extraction in acetonitrile. J. Braz. Chem. Soc., 2003, 14, 577-583

[7] Chicharro, M; Zapardiel, A; Bermejo, E.; Moreno, M. Determination of 3-amino-1,2,4-triazole (amitrole) in environmental waters by capillary electrophoresis. Talanta, 2003, 59, 37 45 .

[8] Barek, J.; Fogg, A. G.; Moreira, J. C.; Zanoni, M. V. B.; Zima, J Polarographic and voltammetric determination of selected triazinebased azo dyes with different reactive groups. Anal. Chim. Acta., 1996, 320,31 . 
[9] Walker, R. Benzotriazole a corrosion inhibitor for antiques some practical surface chemistry. J. Chem. Educ., 1980, 57, 789-791.

[10] Satpati, A. K.; Ravindran, P. V. Electrochemical study of the inhibition of corrosion of stainless steel by 1,2,3-benzotriazole in acidic media. Mat. Chem. Phys., 2008, 109, 352-359.

[11] Satpati, A. K.; Palrecha, M. M.; Sundaresan, R. I. Electrochemical study of the mechanism of interaction of 1,2,3-benzotriazole on SS304 surface in $\mathrm{HCl}$ medium. Ind. J. Chem. Tech., 2008, 15, 163167.

[12] Elbakri, M.; Touir, R.; Touhami, M. E.; Srhiri, A. Benmessaoud, Electrosynthesis of adherent poly(3-amino-1,24-triazole) films on brass prepared in nonaqueous solvents. Corrosion Sci., 2008, 50, 1538-1545.

[13] Jbarah, A. A.; Banert, K.; Holze, R. A. Spectroelectrochemical study of the electrosorption of 4-isopropylsulfanylmethyl-1,2,3triazole on gold. Vib. Spectrosc., 2007, 44, 142-153.

[14] Jbarah, A. A.; Ihle, A.; Banert, K.; Holze, R. A. The electrosorption of 1,2,3-triazole on gold as studied with surface-enhanced Raman spectroscopy. J. Raman. Spectrosc., 2006, 37, 123-131.
[15]

Bard, A. J.; Faulkner, R. L. Electrochemical methods fundamentals and applications, (Wiley, New York) 1980, p. 218.

[16] Nicholson, R. S.; Shain, I. Theory of stationary electrode polarography single scan and cyclic methods applied to reversible irreversible, and kinetic systems. Anal. Chem., 1964, 36,706-723.

[17] Stradyn, Ya. P.; Kadysh, V. P.; Giller, S. A. Polarography of heterocyclic compounds. I. General data on the polarographic behavior of heterocycles and electrochemical reduction of heteroaromatic compounds. Khim. Geterotsikl. Soedin, 1973, 12, $1587-1603$

[18] Bremmel, K.E.; Jameson, G.J.; Biggs, S. Adsorption of ionic surfactants in particulate systems: flotation, stability, and interaction forces. Colloids Surf., A. 1999, 146, 75-87.

[19] Qadeer, R.; Rehan, A. H. A study of the adsorption of phenol by activated carbon from aqueous solution. Turk. J. Chem., 2002, 26, 357-361.

Received: January 15, 2010

Revised: May 14, 2010

Accepted: June 14, 2010

(C) Lokesh et al.; Licensee Bentham Open.

This is an open access article licensed under the terms of the Creative Commons Attribution Non-Commercial License (http://creativecommons.org/licenses/by-nc/3.0/) which permits unrestricted, non-commercial use, distribution and reproduction in any medium, provided the work is properly cited. 